\title{
Renormalization of Non Polynomial Lagrangians in Jaffe's Class
}

\author{
H. EPSTEIN \\ I.H.E.S., Bures-surYvette \\ V. GLASER \\ CERN, Geneva
}

Received April 21, 1971

\begin{abstract}
It is shown how a renormalized perturbation series can be defined for a theory with strictly local, non-polynomial, interacting Lagrangian

$$
\mathscr{L}(x)=\sum_{r=0}^{\infty} t_{r} \frac{: A(x)^{r}:}{r !}
$$
\end{abstract}

so as to preserve locality at every order.

\section{Introduction}

In Ref. [1] an inductive construction of the perturbation series for polynomial Lagrangians was given. It was shown that, given a Wick polynomial $\mathscr{L}(x)$ in a free field $A(x)$ one can construct (by induction on $n$ ) the chronological products

$$
T\left(x_{1}, \ldots, x_{n}\right)=T\left(\mathscr{L}\left(x_{1}\right) \ldots \mathscr{L}\left(x_{n}\right)\right)
$$

which generate the perturbation series of a theory whose interaction Lagrangian reduces to $\mathscr{L}(x)$ in the first order. In this note we show how to extend this method to the case when $\mathscr{L}(x)$ is no longer a Wick polynomial but an entire function in the free field, denoted

$$
\mathscr{L}(x)=\sum_{r=0}^{\infty} t_{r} \frac{: A(x)^{r}:}{r !} .
$$

Such an entire function is still a strictly localized field in the sense of Jaffe [2] provided the coefficients $t_{r}$ do not grow too fast; in fact we shall restrict ourselves to a special class of theories in which

$$
\left|t_{r}\right|<E M^{r} r^{\lambda r}
$$


where $0<\lambda<\frac{1}{2}$. Under such conditions the two point function

$$
(\Omega, \mathscr{L}(x) \mathscr{L}(0) \Omega)=\int e^{-i p x} \varrho(p) d p
$$

is given by

$$
\varrho(p)=\sum_{r=0}^{\infty} \frac{\left|t_{r}\right|^{2}}{r !} \varrho_{r}(p)
$$

where $\varrho_{r}(p)$ is the $r$-particle phase space and, for $r \geqq 2$, is bounded by

$$
\frac{C^{r} p^{2(r-2)}}{(r !)^{2}} \text {, hence } \sum_{r=2}^{\infty} \frac{M^{\prime r} p^{2(r-2)}}{(r !)^{(3-2 \lambda)}} \leqq E^{2} \exp \left(M^{\prime \prime}|p|^{\frac{1}{2}-\lambda}\right) \text {. }
$$

As a consequence $\varrho(p)$ can be integrated with test functions decreasing like $\exp \left(-A|p|^{\frac{1}{\sigma}}\right)$ where $\frac{1}{\sigma}>\frac{1}{\frac{3}{2}-\lambda}$, i.e. $\sigma<\frac{3}{2}-\lambda$.

Such functions may have Fourier transforms with compact support only if $\sigma>1$. Hence the condition $\lambda<\frac{1}{2}$ ensures the strict locality of $\mathscr{L}(x)$. In the inductive construction of the perturbation series, we wish to preserve this strict locality, i.e. to satisfy the causal factorization property of the chronological products. For detailed information regarding generalized functions, see [2-6].

\section{Induction Hypothesis}

Just as in the polynomial case, it is useful to introduce the local fields

$$
\mathscr{L}^{(r)}(x)=\sum_{s=0}^{\infty} t_{r+s} \frac{: A(x)^{s}:}{s !}
$$

and to define chronological products for all the $\mathscr{L}^{(r)}$. These will be denoted $T_{r}(X)$ where $X$ stands for $\left(x_{1}, \ldots, x_{v}\right) \in \mathbb{R}^{4 v}$ and $r=\left(r_{1}, \ldots, r_{v}\right)$ is a multiinteger. These notations are the same as in Ref. [1] and will not be further explained. In our induction hypothesis we assume that the $T_{r}(X)$ have been constructed for all $X$ with $|X| \leqq n-1$ and satisfy

$$
T_{r}(X)=\sum_{s} t_{r+s}(X) \frac{: A(X)^{s}:}{s !}
$$

Here the summation runs over all positive multi-integers $s=\left(s_{1}, \ldots, s_{v}\right)$ $(X=1, \ldots, v) ;: A(X)^{s}:$ means $: A\left(x_{1}\right)^{s_{1}} \ldots A\left(x_{v}\right)^{s_{v}}:$ and as usual $s !=\prod_{j=1}^{v}\left(s_{j} !\right)$. The $t_{r}(X)=\left(\Omega, T_{r}(X) \Omega\right)$ are translationally invariant generalized functions which satisfy

$$
\left|\left\langle t_{r}, f\right\rangle\right| \leqq E_{|X|} M_{|X|}^{|r|}|r|^{\hat{\lambda}|r|}\|f\|_{|X|} .
$$


Here $M_{|X|}$ and $E_{|X|}$ are constants depending only on $|X|$ and

$$
\begin{gathered}
\|f\|_{|X|}=\sum_{\alpha} \sup (1+|x|)^{P}|\alpha|^{-\sigma|\alpha|} A^{-|x|}\left|D^{\alpha} f(x)\right| \\
\equiv N(f ; \sigma, A, P) .
\end{gathered}
$$

Here $\alpha$ runs over all multi-indices; The positive constants $P, A, \sigma$ depend only on $|X|$ (and not on $r$ or $\alpha$ ) and

$$
1<\sigma<\frac{3}{2}-\lambda .
$$

We also make the same assumption for the antichronological products (see [1]) denoted

$$
\bar{T}_{r}(X)=\sum_{r} \bar{t}_{r}(X) \frac{: A(X)^{r}:}{r !}
$$

The $\bar{t}_{r}(X)$ are supposed to satisfy exactly the same inequalities (5), (6) as the $t_{r}(X)$. In the following $T_{r}{ }^{\#}(X)$ and $t_{r}{ }^{*}(x)$ stand for $T_{r}(X)$ or $\bar{T}(X)$ and for $t_{r}(X)$ or $\bar{t}_{r}(X)$.

\section{Going from $n-1$ to $n$ : First Step}

Our first step in showing that the inductive construction extends the property (5) to the case $|X|=n$ is to show that

$$
\left(\Omega, T_{r-s}^{\#}(X) T_{s}^{\#}\left(X^{\prime}\right) \Omega\right) \quad\left(\text { where }|X|+\left|X^{\prime}\right|=n\right)
$$

satisfies a condition of the type (5). More generally let

$$
X=\left(x_{1}, \ldots, x_{v}\right), \quad X^{\prime}=\left(x_{1}^{\prime}, \ldots, x^{\prime}{ }_{x}\right)
$$

with $v$ and $x \leqq n-1, r=\left(r_{1}, \ldots, r_{v}\right), s=\left(s_{1}, \ldots, s_{x}\right)$ two multi-indices. Then $\left(\Omega, T_{r}^{\#}(X) T_{s}^{\#}\left(X^{\prime}\right) \Omega\right)$

$$
=\sum_{l=0}^{\infty} \sum_{\substack{a, b \\|a|=|b|=l}} t_{r+a}^{\#}(X) t_{\mathrm{s}+b}\left(X^{\prime}\right) \frac{\left(\Omega,: A(X)^{a}:: A\left(X^{\prime}\right)^{b}: \Omega\right)}{a ! b !} .
$$

It is easy to see (by "separating the points" in : $A(X)^{a}:$ and $: A\left(X^{\prime}\right)^{b}:$ ) that $\frac{1}{a ! b !} t_{r+a}^{\#}(X) t_{s+b}^{\#}\left(X^{\prime}\right)\left(\Omega,: A(X)^{a}:: A\left(X^{\prime}\right)^{b}: \Omega\right)$ is the sum of $l !$ Terms, each of which is of the form

$$
\frac{1}{a ! b !} t_{r+a}^{\#}(X) t_{s+b}^{\#}\left(X^{\prime}\right) \prod_{j=1}^{l}\left(\Omega, A\left(x_{u(j)}\right) A\left(x_{v(j)}^{\prime}\right) \Omega\right) .
$$

Here $u$ is a map from $(1, \ldots, l)$ into $(1, \ldots, v)$ taking exactly $a_{j}$ times the value $j$ for each $j=1, \ldots, v ; v$ is a map from $(1, \ldots, l)$ into $(1, \ldots, x)$ taking 
$b_{j}$ times the value $j$ for each $j=1, \ldots, \varkappa$. To study this quantity we use the variables

$\xi_{j}=x_{j}-x_{v} \quad\left(j=1, \ldots, v-1\right.$. Conventionally we also introduce $\left.\xi_{v}=0\right)$

$\xi_{j}^{\prime}=x_{j}^{\prime}-x_{\varkappa}^{\prime} \quad\left(j=1, \ldots, x-1 ; \xi_{x}^{\prime} \equiv 0\right)$

$\eta=x_{v}-x_{x}^{\prime}$.

If we integrate (9) with a test function $f\left(\xi, \xi^{\prime}, \eta\right)$ we obtain

$$
\begin{gathered}
\frac{1}{a ! b !} \int t_{r+a}^{\#}(\xi) t_{s+b}^{\#}\left(\xi^{\prime}\right) \psi\left(\xi, \xi^{\prime}\right) d \xi d \xi^{\prime}, \\
\psi\left(\xi, \xi^{\prime}\right)=\int f\left(\xi, \xi^{\prime}, \eta\right) d \eta \exp -i\left[P \eta+\sum_{j=1}^{v} \xi_{j} p_{I_{J}}+\sum_{j=1}^{x} \xi_{j}^{\prime} p_{J_{J}}\right] \\
d^{4} P \delta\left(P-\sum_{j=1}^{l} p_{j}\right) \prod_{j=1}^{l} \delta\left(p_{j}^{2}-m^{2}\right) \theta\left(p_{j}^{0}\right) d^{4} p_{j} .
\end{gathered}
$$

Here

$$
p_{I_{J}}=\sum_{u(t)=j} p_{t}, \quad p_{J_{J}}=\sum_{v(t)=j} p_{t}
$$

and we recall that $\xi_{v}=\xi_{x}^{\prime}=0$. We also denote

$$
\hat{p}=\left(p_{I_{1}}, \ldots, p_{I_{v-1}}\right), \quad \check{p}=\left(p_{J_{1}}, \ldots, p_{J_{\varkappa-1}}\right) .
$$

According to the induction hypothesis, there are positive constants $C, P, Q, \sigma, L, F$ independent of $r, s, a, b, u, v$ (i.e., depending only on $n$ ) such that the modulus of (10) is bounded by

$$
\begin{gathered}
\frac{F}{a ! b !} L^{|r|+|s|+|a|+|b|}|r+a|^{\lambda|r+a|}|S+b|^{\lambda|s+b|} \\
\sum_{\alpha, \beta} C^{|\alpha|+|\beta|} \sup _{\circlearrowright}(1+|\xi|)^{P}\left(1+\mid \xi^{\prime}\right)^{Q}|\alpha|^{-\sigma|\alpha|}|\beta|^{-\sigma|\beta|}\left|D_{\xi}^{\alpha} D_{\xi^{\prime}}^{\beta} \psi\left(\xi, \xi^{\prime}\right)\right| .
\end{gathered}
$$

But

$$
\begin{gathered}
D_{\breve{\xi}}^{\alpha} D_{\xi^{\prime}}^{\beta} \psi=\sum_{\substack{\gamma, \mu \\
\gamma \leq x \\
\mu \leqq \beta}} \frac{\alpha !(\alpha-\gamma) !}{\mu !(\beta-\mu) !} \\
\int B_{\gamma, \mu}^{l}\left(\xi, \xi^{\prime}, \eta\right)\left(-i \frac{\partial}{\partial \eta_{0}}+1\right)^{2}\left(-i \frac{\partial}{\partial \eta^{0}}\right)^{M} D_{\xi}^{\alpha-\gamma} D_{\xi^{\prime}}^{\beta-\mu} f\left(\xi, \xi^{\prime}, \eta\right) d \eta
\end{gathered}
$$

where $M=|\gamma|+|\mu|+2 l-1$ and

$$
\begin{aligned}
B_{\gamma, \mu}^{l}\left(\xi, \xi^{\prime}, \eta\right)= & \int d^{4} P \frac{(i \hat{p})^{\gamma}(i \breve{p})^{\mu}}{\left(P^{0}+1\right)^{2}\left(P^{0}\right)^{M}} e^{-i P \eta-i \hat{p}, \xi-i^{\vee}, \xi^{\prime}} \\
& \delta\left(P-\sum_{j=1}^{l} p_{j}\right) \prod_{j=1}^{l} \delta\left(p_{j}^{2}-m^{2}\right) \theta\left(p_{j}^{0}\right) d^{4} p_{j} .
\end{aligned}
$$


It is straightforward to verify (see e.g. a similar calculation in [1]) that there is a universal constant $A$ such that

$$
\left|B_{\gamma, \mu}^{l}\left(\xi, \xi^{\prime}, \eta\right)\right| \leqq \frac{A^{l}}{(l !)^{2}} \quad(l \geqq 1)
$$

(The case $l=0$ is trivial.) The modulus of $(10)$ is bounded by

$$
\begin{gathered}
\frac{F A^{l}}{(l !)^{2} a ! b !} L^{|r|+|a|+|s|+|b|}|r+a|^{\lambda|r+a|}|s+b|^{|\lambda| s+b \mid} \\
\sum_{\alpha, \beta} C^{|\alpha|+|\beta|}|\alpha|^{-\sigma|\alpha|}|\beta|^{-\sigma|\beta|} \sup _{\xi, \xi^{\prime}}(1+|\xi|)^{P}\left(1+\left|\xi^{\prime}\right|\right)^{Q} \\
\int d \eta \frac{\alpha !}{\gamma !(\alpha-\gamma) !} \frac{\beta !}{\mu !(\beta-\mu) !} \\
\cdot\left|\left(-i \frac{\partial}{\partial \eta^{0}}+1\right)^{2}\left(-\frac{i \partial}{\partial \eta^{0}}\right)^{|\gamma|+|\mu|+2 l-1} D_{\xi}^{\alpha-\gamma} D_{\xi^{\prime}}^{\beta-\mu} f\left(\xi, \xi^{\prime}, \eta\right)\right| .
\end{gathered}
$$

We note the following inequalities:

1. if $x$ is a positive real number

$$
e^{-x} x^{x} \leqq \Gamma(x+1) \leqq 3 x^{x} .
$$

2. let $\alpha_{1}, \ldots, \alpha_{N}$ be integers $\geqq 0$ with $|\alpha|=\sum_{j=1}^{N} \alpha_{j}=\omega$. Then

so that

$$
\sum_{\substack{x \\|\alpha|=\omega}} \frac{\omega !}{\alpha !}=N^{\omega}
$$

$$
\begin{gathered}
\sum_{\substack{\alpha \\
|\alpha|=\omega}} 1 \leqq N^{\omega} ; \frac{\omega !}{\alpha !}<N^{\omega} ; \\
\prod_{j=1}^{N} \alpha_{j}^{\sigma \alpha_{j}} \leqq \alpha^{\sigma \alpha} \leqq \omega^{\sigma \omega} \leqq N^{\sigma \omega} \alpha^{\sigma \alpha} \quad(\text { all } \sigma>0) .
\end{gathered}
$$

By using these inequalities it is easy to verify that there are constants $B_{v+\varkappa}, L_{v+\varkappa}, C_{v+\varkappa}^{\prime}$ depending on $v+\varkappa$ but independent of $r, s, a, b, u, v, l$, such that

$$
\begin{aligned}
& \left|\frac{1}{a ! b !}\left\langle t_{r+a} \otimes t_{s+b}, \psi\right\rangle\right| \\
& \leqq F \frac{L_{v+x}^{r|+| s \mid+1} B_{v+x}^{l}(2 l+1)^{\sigma(2 l+1)}(|r|+l)^{\lambda(|r|+l)}(|s|+l)^{\lambda(|s|+l)}}{(l !)^{2} a ! b !}\|f\|_{v+x}
\end{aligned}
$$

and

$$
\|f\|_{v+\chi}=\sum_{\gamma} \sup _{\xi, \xi^{\prime} \eta} C^{\prime|\alpha|}|\alpha|^{-\sigma|\alpha|}\left|D^{\alpha} f\left(\xi, \xi^{\prime}, \eta\right)\right|\left(1+\left|\xi, \xi^{\prime}, \eta\right|\right)^{P+Q+5} .
$$



as (9))

As a consequence we have (remembering that there are $l$ ! terms such $\int\left(\Omega, T_{r}^{\#}(X) T_{s}^{\#}\left(X^{\prime}\right) \Omega\right) f\left(\xi, \xi^{\prime}, \eta\right) d \xi d \xi^{\prime} d \eta \mid$

$$
\leqq F\|f\|_{v+x} \sum_{l=0}^{\infty} L_{v+x}^{|r|+|s|} B_{v+x}^{\prime l} e^{-(3-2 \lambda-2 \sigma) l \log l}|r|^{\lambda|r|}|s|^{\lambda|s|}
$$

where $F, B_{v+\varkappa}^{\prime}$ and $L_{v+\varkappa}^{\prime}$ are constants independent of $r$ and $s$ (and $l$ ). If $3-2 \lambda-2 \sigma>0$, there are constants $R$ and $U$ depending on $v+x$ but independent of $r$ and $s$ such that

$$
\begin{aligned}
& \left|\int\left(\Omega, T_{r}^{\#}(X) T_{s}^{\#}\left(X^{\prime}\right) \Omega\right) f\left(\xi, \xi^{\prime}, \eta\right) d \xi d \xi^{\prime} d \eta\right| \\
& \quad \leqq F R^{|r|+|s|}(|r|+|s|)^{\lambda(|r|+|s|)} N(f, \sigma, U, P+Q+5) .
\end{aligned}
$$

Moreover, by iterating this process we reach the following conclusion: Let $X=x_{1}, \ldots, x_{n}$ and let $I_{1}, \ldots, I_{v}$ be a partition of $X$ into non empty subsets such that each of them contains at most $n-1$ points. Let $r=\left(r_{1}, \ldots, r_{n}\right)$ be a multi-integer and $r\left|I_{1}, \ldots, r\right| I_{v}$ be its restriction to $I_{1}, \ldots, I_{v}$ in the obvious sense. There exist constants $F_{n}, K_{n}, U_{n}$ and $P_{n}$ depending only on $n$, and not on $r$ (or $v$ ) such that

$$
\begin{gathered}
\left|\int\left(\Omega, T_{r \mid I_{1}}^{\#}\left(I_{1}\right) T_{r \mid I_{2}}^{\#}\left(I_{2}\right) \ldots T_{r \mid I_{v}}^{\#}\left(I_{v}\right) \Omega\right) f\left(\xi_{1}, \ldots, \xi_{n-1}\right) d \xi_{1} \ldots d \xi_{n-1}\right| \\
\leqq F_{n} K_{n}^{|r|}|r|^{\lambda|r|} \quad N\left(f, \sigma, U_{n}, P_{n}\right) .
\end{gathered}
$$

(Here $\xi_{j}=x_{j}-x_{n}$.)

Let $X=\left(x_{1}, \ldots, x_{n}\right), Y=\left(x_{1}, \ldots, x_{n-1}\right)$. According to the procedure of [1] we define

$$
\begin{gathered}
D\left(Y ; x_{n}\right)=\sum_{\substack{I \cup J=Y \\
I \cap J=\emptyset \\
I \neq \emptyset}}(-1)^{|I|}[T(J, n), \bar{T}(I)] \\
D\left(Y ; x_{n}\right)=\sum_{r} d_{r}(Y ; n) \frac{: A(X)^{r}:}{r !} \\
d_{r}(Y ; n)=\sum_{\substack{I \cup J=Y \\
I \cap J=\emptyset \\
I \neq \emptyset}}(-1)^{|I|}\left(\Omega,\left[T_{r \mid(J, n)}(J, n), \bar{T}_{r \mid I}(I)\right] \Omega\right)
\end{gathered}
$$

with obvious notations. It follows that

$$
\left|\int d_{r}(Y ; n) f(\xi) d \xi\right| \leqq F_{n} K_{n}^{\prime|r|}|r|^{\hat{\lambda}|r|} N\left(f, \sigma, U_{n}, P_{n}\right)
$$

We note that the preceding estimates actually serve to define the operator-valued distributions of the form

$$
G(X)=\sum_{r} g_{r}(X) \frac{: A(X)^{r}:}{r !}
$$


where the $g_{r}(X)$ are translationally invariant generalized functions satisfying:

$$
\left|\int g_{r}(X) f(X) d X\right| \leqq B C^{|r|}|r|^{\lambda|r|} N(f, \sigma, A, P)
$$

(where $B, C, A, P, \sigma$ may depend on $G$ but not on $r$, and $1<\sigma<\frac{3}{2}-\lambda$ ). (Note that, in particular, we could take all but a finite number of the $g_{r}(X)$ to be identically 0 .) We also denote

$$
\begin{aligned}
G_{r}(X) & =\sum_{s} g_{r+s}(X) \frac{: A(X)^{s}:}{s !}, \\
G_{r}{ }^{(N)}(X) & =\sum_{|s| \leqq N} g_{r+s}(X) \frac{: A(X)^{s}:}{s !} .
\end{aligned}
$$

Then estimates identical to the preceding show that, if $G, H, \ldots, K$ are $p$ objects similar to $G$

$$
\begin{gathered}
\lim _{N_{1}, \ldots, N_{p} \rightarrow \infty} \int G^{\left(N_{1}\right)}\left(X_{1}\right) H^{\left(N_{2}\right)}\left(X_{2}\right) \ldots K^{\left(N_{p}\right)}\left(X_{p}\right) \Omega \\
f\left(X_{1}, \ldots, X_{p}\right) d X_{1} \ldots d X_{p}
\end{gathered}
$$

exists in the sense of the strong topology of the Hilbert space provided some $N(f, \sigma, A, P)$ is finite. As a consequence the $G_{r}(X)$ define operator valued generalized functions on a dense domain. On this domain they can be freely multiplied. The limiting procedure shows that these operators are generalized functions in the Jaffe class, and that the considerations of locality, supports etc. usual in the polynomial case also apply to them.

As a consequence by the same arguments as in [1], the support of $D_{r}(Y, n)$ (hence of $\left.d_{r}(Y, n)\right)$ is contained in $\Gamma^{+} \cup \Gamma^{-}$,

$$
\Gamma^{+}=\left\{x_{1}, \ldots, x_{n}: x_{j}-x_{n} \in \bar{V}^{+} \text {for all } j\right\}=-\Gamma^{-}
$$

and the next problem is to split $D(Y, n)$ into two pieces with supports $\Gamma^{+}$and $\Gamma^{-}$, by splitting each $d_{r}(Y, n)$. In so doing we must be able to have each of the two pieces satisfy an inequality of the type (18). We follow exactly the same procedure as in the polynomial case (see [1]).

\section{Second Step : The Splitting Operation}

Let $\tau>0$ be an arbitrarily small number. Then there exists a function III (indeed an infinity of such functions) with the following properties:

1. IU is defined over $\mathbb{R}^{N}$ and is $\mathscr{C}^{\infty}$ everywhere except at the origin.

2. $\mathrm{III}(\varrho \xi)=\mathrm{III}(\xi)$ for every $\xi \neq 0$ in $\mathbb{R}^{N}$ and every $\varrho>0$.

3. $0 \leqq \amalg \leqq 1$; IU takes the value 1 (resp. the value 0 ) in a neighbourhood of $\Gamma^{+-}\{0\}$ (resp. in a neighbourhood of $\Gamma^{--}\{0\}$ ). 
4. There exists a constant $C$ such that for all $\xi \neq 0$ and all $\alpha$, $\left|D^{\alpha} \amalg(\xi)\right| \leqq C|\xi|^{-|\alpha|}|\alpha|^{(\tau+1)|\alpha|}$.

To construct such a function, one first constructs it on the unit sphere and then extends it by homogeneity to the whole of $\mathbb{R}^{N_{-}}\{0\}$.

According to (18), $d_{r}$ is a continuous linear functional on the Banach space of $\mathscr{C}^{\infty}$ functions $f$ such that $N\left(f, \sigma, U_{n}, P_{n}\right)<\infty$. We plan to show that, provided $\tau$ has been chosen small enough, there exist new constants $U_{n}^{\prime}, U_{n}^{\prime \prime}, R_{n}, S_{n}$ such that

(i) if $f$ is $\mathscr{C}^{\infty}$ with $D^{\alpha} f(0)=0$ for all $\alpha$ and $N\left(f, \sigma-\tau, U_{n}^{\prime \prime}, P_{n}\right)<\infty$, then $\amalg f$ is a $\mathscr{C}^{\infty}$ function such that

$$
N\left(\amalg f, \sigma, U_{n}, P_{n}\right) \leqq R_{n} N\left(f, \sigma-\tau, U_{n}^{\prime \prime}, P_{n}\right) .
$$

(ii) There exists an operator $W$ on $\mathscr{C}^{\infty}$ functions $f$ satisfying $N\left(f, \sigma-2 \tau, U_{n}^{\prime}, \mathrm{P}_{n}\right)<\infty$ such that $W f$ is again a $\mathscr{C}^{\infty}$ function and:

a) $N\left(W f, \sigma-\tau, U_{n}^{\prime \prime}, P_{n}\right) \leqq S_{n} N\left(f, \sigma-2 \tau, U_{n}^{\prime}, P_{n}\right)$.

b) $D^{\alpha}(W f)(0)=0$ for all $\alpha$ (for any $f$ ).

c) If $D^{\alpha} f(0)=0$ for all $\alpha$ then $W f=f$.

From this it will follow that:

$$
N\left(\amalg W f, \sigma, U_{n}, P_{n}\right) \leqq R_{n} S_{n} N\left(f, \sigma-2 \tau, U_{n}^{\prime}, P_{n}\right)
$$

which will enable us to define the advanced "function" $a_{r}(Y ; n)$ by

with

$$
\left\langle a_{r}, f\right\rangle=\left\langle d_{r}, \amalg W f\right\rangle
$$

$$
\left|\left\langle a_{r}, f\right\rangle\right| \leqq F_{n}^{\prime} K_{n}^{\prime \prime|r|}|r|^{\lambda|r|} N\left(f, \sigma-2 \tau, U_{n}^{\prime}, P_{n}\right) .
$$

This will yield a set of $t_{r}(X)$ for $|X|=n$ again satisfying

$$
\left|\left\langle t_{r}, f\right\rangle\right| \leqq E_{n} M_{n}^{|r|}|r|^{\lambda|r|} N\left(f, \sigma-2 \tau, U_{n+1}, P_{n}\right) .
$$

Proof of (i). We assume that $\tau$ has been chosen so that $0<\tau<\frac{\sigma-1}{2}$. Suppose $f$ is a $\mathscr{C}^{\infty}$ function with $D^{\alpha} f(0)=0$ for all $\alpha$ and $N\left(f, \sigma-\tau, U_{n}^{\prime \prime}, P_{n}\right) \leqq 1$, where $U_{n}^{\prime \prime} \leqq 1$. (Note that $\sigma-\tau>\tau+1>1$.) We have:

$$
D^{\alpha} f(\xi)=\int_{0}^{1} d t(1-t)^{\omega-1} \sum_{\substack{\gamma \\|,|=\omega}} \frac{\omega}{\gamma !} \xi^{\gamma} D^{\alpha+\gamma} f(t \xi) .
$$

Hence, for $|\xi|<1$,

$$
\left|D^{\alpha} f(\xi)\right| \leqq U_{n}^{\prime \prime}|\alpha|+\omega(|\alpha|+\omega)^{(\sigma-\tau)(|\alpha|+\omega)} \frac{N^{\omega}}{\omega !}|\xi|^{\omega} .
$$


For $\xi \neq 0$

$$
\begin{aligned}
\left|D^{\alpha}(\mathrm{II} f)(\xi)\right| & =\left|\sum_{\gamma \leqq \alpha} \frac{\alpha !}{\gamma !(\alpha-\gamma) !} D^{\gamma} \operatorname{III}(\xi) D^{\alpha-\gamma} f(\xi)\right| \\
& \leqq \sum_{\gamma \leqq \alpha} \frac{\alpha !}{\gamma !(\alpha-\gamma) !} C \frac{|\gamma|^{(1+\tau)|\gamma|}}{|\xi|^{|\gamma|}}\left|D^{\alpha-\gamma} f(\xi)\right| .
\end{aligned}
$$

For $|\xi| \leqq 1$, by $(23)$, this is smaller than

While, for $|\xi| \geqq 1$,

$$
C|\alpha|\left(e N^{2}\right)^{|\alpha|} U_{n}^{\prime \prime|\alpha|}|\alpha|^{\sigma|\alpha|} .
$$

$$
\begin{aligned}
(1+|\xi|)^{P_{n}} & \mid D^{\alpha}(\text { III } f)(\xi) \mid \\
& \leqq \sum_{\gamma \leqq \alpha} \frac{\alpha !}{\gamma !(\alpha-\gamma) !} C|\gamma|^{(1+\tau)|\gamma|} U_{n}^{\prime \prime|\alpha-\gamma|}|\alpha-\gamma|^{(\sigma-\tau)|\alpha-\gamma|} \\
& \leqq \sum_{\omega \leqq|\alpha|} C N^{\omega} \frac{U^{\prime \prime|\alpha|-\omega}}{(|\alpha|-\omega)^{\tau(|\alpha|-\omega)} \omega^{(\sigma-\tau-1) \omega}}|\alpha|^{\sigma|\alpha|} \\
& \leqq \frac{|\alpha| C(2 N)^{|\alpha|}}{|\alpha|^{\tau|\alpha|}}|\alpha|^{\sigma|\alpha|} .
\end{aligned}
$$

Finally, we see that there is a constant $C_{1}$, independent of $\alpha$ and $U_{n}^{\prime \prime}$, such that

$$
\begin{aligned}
\sup _{\xi}(1+|\xi|)^{P_{n}}\left|D^{\alpha}(I I f)(\xi)\right| & \\
& \leq C_{1}^{(1+|\alpha|)}\left(U_{n}^{\prime \prime|\alpha|}+|\alpha|^{-\tau|\alpha|}\right)|\alpha|^{\sigma|\alpha|} .
\end{aligned}
$$

Hence it is obviously possible to choose $U_{n}^{\prime \prime}$ sufficiently small so that the series

$$
\sum_{\alpha} C_{1}\left(C_{1} U_{n}^{-1}\right)^{|\alpha|}\left(U_{n}^{\prime \prime|\alpha|}+|\alpha|^{-\tau|\alpha|}\right)
$$

converges to a constant $R_{n}$. This proves (i).

Proof of (ii). To prove (ii) we choose a $\mathscr{C}^{\infty}$ function $w$ over $\mathbb{R}^{N}$ such that $w(\xi)=1$ if $|\xi| \leqq \frac{1}{2}, w(\xi)=0$ if $|\xi| \geqq 1$ and, for all $\alpha$

$$
D^{\alpha} w(\xi)<K|\alpha|^{\left(1+\frac{r}{2}\right)|\alpha|}
$$

We define the operator $W$ by

$$
(W f)(\xi)=f(\xi)-\sum_{\alpha} w\left(\varepsilon_{|\alpha|}^{-1} \xi\right) \frac{\xi^{\alpha}}{\alpha !} D^{\alpha} f(0) .
$$

Here $\left\{\varepsilon_{k}\right\}$ is a decreasing sequence of positive real numbers $<1$ satisfying:

$$
\varepsilon_{k}=\varepsilon k^{-(\sigma-2 \tau-1)} \text { for all integers } k>0 \text {. }
$$


Here $\varepsilon$ is a real number $>0$.

$$
\begin{aligned}
& D^{\alpha}[(1-W) f](\xi)=\sum_{\beta} \sum_{\gamma} \varepsilon_{|\beta|}^{-|\alpha|+|\gamma|}\left(D^{\alpha-\gamma} w\right)\left(\frac{\xi}{\varepsilon_{|\beta|}}\right) \frac{\xi^{\beta-\gamma}}{(\beta-\gamma) !} D^{\beta} f(0) \\
& \eta \leqq \beta \\
& \left|D^{\alpha}(1-W) f(\xi)\right| \leqq \sum_{\beta} \sum_{\gamma} \delta|\beta|^{|\beta|} K|\alpha| \alpha-\gamma\left|\left(1+\frac{\tau}{2}\right)\right| \alpha-\gamma\left|N^{|\beta-\gamma|}\right| \beta-\left.\gamma\right|^{-|\beta-\gamma|} \\
& \begin{array}{l}
\gamma \leqq \beta \\
\gamma \leqq \alpha
\end{array} \\
& U_{n}^{\prime|\beta|}|\beta|^{(\sigma-2 \tau)|\beta|} .
\end{aligned}
$$

The sum of terms for which $|\beta|>|\alpha|$ is majorized by:

$$
\begin{aligned}
\sum_{\substack{\beta \\
|\beta| \leqq|\alpha|}} \sum_{\substack{\gamma \leqq \alpha \\
\gamma \leqq \beta}}\left\{\varepsilon(|\beta|-|\alpha|)^{-(\sigma-2 \tau-1)}\right\}^{(|\beta|-|\alpha|)} K N^{|\beta|-|\gamma|} \\
\cdot|\alpha-\gamma|^{\tau / \alpha-\gamma \mid}(|\beta|-|\alpha|)^{(\sigma-2 \tau-1)(|\beta|-|\alpha|)} U_{n}^{\prime|\beta|-|\alpha|} \\
\cdot U_{n}^{\prime|\alpha|}|\alpha|^{(\sigma-2 \tau)|\alpha|} 2^{(\sigma-2 \tau)|\beta|} \\
\leqq K|\alpha| U_{n}^{\prime|\alpha|}|\alpha|^{\left(\sigma-\frac{3}{2} \tau\right)|\alpha|} 2^{(\sigma-2 \tau)|\alpha|} \\
\sum_{m=0}^{\infty} N^{2 m} U_{n}^{\prime m} 2^{(\sigma-2 \tau) m} \varepsilon^{m} .
\end{aligned}
$$

If $U_{n}^{\prime}$ is small enough, this is bounded by

$$
\text { const } U_{n}^{\prime|\alpha|} 2^{(\sigma-2 \tau)|\alpha|}|\alpha|^{\left(\sigma-\frac{3}{2} \tau\right)|\alpha|+1} \text {. }
$$

The sum of terms for which $|\alpha| \geqq|\beta|$ is majorized by

$$
\begin{aligned}
& \sum_{\substack{\beta \\
|\beta| \leqq|\alpha|}} \sum_{\substack{\gamma \leqq \alpha \\
\gamma \leqq \beta}} K|\beta|^{(\sigma-2 \tau-1)(|\alpha|-|\beta|)}|\alpha|^{\left(1+\frac{\tau}{2}\right)|\alpha|}|\gamma|^{-\frac{\tau}{2}|\gamma|} \\
& \cdot 2^{|\beta|} N^{|\beta|-|\gamma|}|\beta|^{(\sigma-2 \tau-1)|\beta|} U_{n}^{\prime|\beta|} \varepsilon^{-|\alpha|} \\
& \leqq \sum_{\substack{\beta \\
|\beta|<|\alpha|}} K|\beta| N^{2|\beta|} 2^{|\beta|}|\alpha|^{\left(\sigma-\frac{3}{2} \tau\right)|\alpha|} U_{n}^{\prime|\beta|} \varepsilon^{-|\alpha|} .
\end{aligned}
$$

If $U_{n}^{\prime} \leqq 1$, as we shall suppose, this is bounded by

$$
K \frac{(2 N)^{3|\alpha|}|\alpha|^{(\sigma-\tau)|\alpha|}}{|\alpha|^{\frac{\tau}{2}|\alpha|} \varepsilon^{|\alpha|}}
$$

Hence, for sufficiently small $U_{n}^{\prime}$, the series

$$
\sum_{\alpha} U_{n}^{\prime \prime-|\alpha|}|\alpha|^{-|\sigma-\tau||\alpha|} \sup _{\xi}(1+|\xi|)^{P_{n}}\left|D^{\alpha}(1-W) f(\xi)\right|
$$

is majorized by a finite constant, independent of $f$. This proves (ii). 
Remark 1. Note that the same splitting operation has been used to define the $a_{r}(Y ; n)$ for all $r$. This operation depends on $n$.

Remark 2. If $f$ is $\mathscr{C}^{\infty}$ and verifies: $D^{\alpha} f(0)=0$ for all $\alpha$, and $N\left(f, \sigma-\tau, U_{n}^{\prime \prime}, P_{n}\right)<\infty$, the quantity $\left\langle d_{r}\right.$, IU $\left.f\right\rangle=\left\langle a_{r}, f\right\rangle$ does not depend on any particular choice of III. Indeed, let III' be another auxiliary function having the properties 1 to 4 required from IU. Let $\left\{f_{k}\right\}$ be a sequence of $\mathscr{C}^{\infty}$ functions such that $N\left(f-f_{k}, \sigma-\tau, U_{n}^{\prime \prime}, P_{n}\right) \rightarrow 0$ as $k \rightarrow \infty$, and that each $f_{k}$ vanishes in a neighbourhood of 0 . Then

$$
\left\langle d_{r}, \amalg f\right\rangle=\lim _{k \rightarrow \infty}\left\langle d_{r}, \amalg f_{k}\right\rangle=\lim _{k \rightarrow \infty}\left\langle d_{r}, \amalg^{\prime} f_{k}\right\rangle=\left\langle d_{r}, \amalg^{\prime} f\right\rangle .
$$

However, the splitting operation depends on the particular choice of the operator $W$, i.e. on the particular choice of the auxiliary function $w$ and of the sequence $\varepsilon_{|\alpha|}$.

Remark 3: ambiguity of the definition of the $a_{r}$; Lorentz invariance. To simplify further consideration let $\sigma^{\prime}=\sigma-2 \tau$ and let $\mathscr{C}^{\prime}{ }_{\sigma^{\prime}}$ be the class of generalized functions $G$ such that there exist constants $K$ and $V$ (depending on $G$ ) such that, for all $f$,

$$
|\langle G, f\rangle| \leqq K N\left(f, \sigma^{\prime}, V, P_{n}\right) .
$$

Let $C$ be a generalized function in the class $\mathscr{C}_{\sigma^{\prime}}^{\prime}$ with

$$
|\langle C, f\rangle| \leqq N\left(f, \sigma, U_{n}, P_{n}\right)
$$

having support in $\Gamma^{+} \cup \Gamma^{-}$. Suppose there are two pairs of generalized functions $F_{1}{ }^{ \pm}, F_{2}{ }^{ \pm}$in the class $\mathscr{C}^{\prime}{ }^{\prime}$, with

$$
\left\{\begin{array}{l}
\text { support } F_{j}^{ \pm} C \Gamma^{ \pm} \\
F_{j}^{+}-F_{j}^{-}=C, \quad j=1,2 .
\end{array}\right.
$$

Then $F_{1}{ }^{+}-F_{2}{ }^{+}=F_{1}{ }^{-}-F_{2}{ }^{-}$is a generalized function in $\mathscr{C}^{\prime}{ }^{\prime}$, with support at the origin. The Fourier transform of a member $G$ of $\mathscr{C}^{\prime}{ }_{\sigma^{\prime}}$ with support at the origin is an entire function (over complex momentum space) $\tilde{G}$ such that

$$
|\tilde{G}(p+i q)|<\text { const } \exp \left(B|p+i q|^{\frac{1}{\sigma^{\prime}}}\right)
$$

and conversely (the constants here depend on $G$ ). The space $\mathscr{C}_{\sigma^{\prime}}^{\prime}$ is invariant under the real Lorentz group and the subspace of its elements having support at 0 is also Lorentz invariant. We denote this subspace by $\mathscr{C}^{\prime \prime}{ }^{\prime}$. 
Suppose $C$ is invariant under the real Lorentz group and $C=F^{+}-F^{-}$ with (support $\left.F^{ \pm}\right) \subset \Gamma^{ \pm}$and $F^{ \pm} \in \mathscr{C}^{\prime}{ }_{\sigma^{\prime}}$. Denote, for each generalized function $G$, and each $\Lambda \in L_{+}^{\uparrow}$,

$$
\langle\Lambda G, f\rangle=\left\langle G, f_{\Lambda}\right\rangle \text { where } f_{\Lambda}(\xi)=f(\Lambda \xi)
$$

(i.e. formally $(\Lambda G)(\xi)=G\left(\Lambda^{-1} \xi\right)$ ). Clearly the mapping $(\Lambda, G) \rightarrow \Lambda G$ is a continuous (and even $\mathscr{C}^{\infty}$ ) map of $L_{+}^{\dagger} \times \mathscr{C}^{\prime}{ }_{\sigma^{\prime}}$ into $\mathscr{C}^{\prime}{ }_{\sigma^{\prime}}$ (the latter being equipped with the topology described previously, i.e. dual of a Fréchet). Let us denote

$$
E(\Lambda)=\Lambda F^{+}-F^{+}=\Lambda F^{-}-F^{-}
$$

This is an element of $\mathscr{C}_{\sigma^{\prime}}^{\prime}$. It satisfies

$$
M E(\Lambda)=E(M \Lambda)-E(M)
$$

for all $\Lambda$ and $M$ in $L_{+}^{\uparrow}$. From this we wish to deduce the existence of an element $E_{0}$ of $\mathscr{C}^{\prime}{ }^{\prime}{ }^{\prime}$ such that ${ }^{1}$ :

$$
E(\Lambda)=\Lambda E_{0}-E_{0}
$$

Then, denoting $G^{ \pm}=F^{ \pm}-E_{0}$ we would find

$$
C=G^{+}-G^{-}, \quad \Lambda G^{ \pm}=G^{ \pm},
$$

i.e. we would have obtained a Lorentz invariant splitting of $C$. However, before doing this we shall require $C$ to possess a property common to all the $d_{r}(Y ; n)$, namely that its Fourier transform should vanish in a real region containing all Jost points. From this it follows that the Fourier transforms $\tilde{F}^{ \pm}$of $F^{ \pm}$are two branches of the same analytic $H(p+i q)$, holomorphic in a domain which is invariant under the whole complex Lorentz group $L_{+}(\mathbb{C})$. Furthermore the Fourier transform $\tilde{E}(\Lambda)$ of $E(\Lambda)$ is the restriction to the reals of the function.

$$
\tilde{E}(\Lambda, p+i q)=H(\Lambda, p+i q)-H(p+i q)
$$

which is entire in $p+i q$ and in $\Lambda \in L_{+}(\mathbb{C})$. It can be shown that, for every complex $\Lambda$, this entire function of $p+i q$ is still of order $\sigma^{\prime-1}$. As a consequence, if we come back to $\xi$-space, we see that $E(\Lambda)$ can be extended to an entire function of $\Lambda \in L_{+}(\mathbb{C})$ with values in $\mathscr{C}^{\prime}{ }_{\sigma^{\prime}}$. Note also that, if $G \in \mathscr{C}^{\prime 0}{ }_{\sigma^{\prime}}, \Lambda G$ is also in this subspace for every $\Lambda \in L_{+}(\mathbb{C})$ since, for real $\Lambda$, $\Lambda G$ is the Fourier transform of $\tilde{G}(\Lambda p)$. Complexifying $\Lambda$ and $p$ in the latter expression again yields an entire function of order $\sigma^{\prime-1}$ which

1 The following considerations are adapted from a paper in preparation in collaboration with $\mathrm{R}$. Stora whom we thank for permission to include them here. 
depends holomorphically on $\Lambda$. Hence $\Lambda G$ is an entire function of $\Lambda$ with values in $\mathscr{C}_{\sigma^{\prime}}^{\prime}$. Let $\mathcal{O}$ denote one of the maximal compact subgroups of $L_{+}(\mathbb{C})$ (for example the subgroup of all complex Lorentz transformations $\Lambda$ such that $\Lambda_{0 j}$ and $\Lambda_{j 0}$ are pure imaginary for $j=1,2,3$, all other $\Lambda_{\mu v}$ being real) and let $d \Lambda$ denote the invariant measure on $\mathcal{O}$ normalized so that $\int_{\mathscr{O}} d \Lambda=1$.

Set

$$
E_{0}=-\int_{\mathscr{O}} E(M) d M .
$$

Since (27) obviously extends to all $\Lambda$ and $M$ in $L_{+}(\mathbb{C})$ by analytic continuation,

$$
\begin{aligned}
E(\Lambda) & -\Lambda E_{0}+E_{0} \\
& =\int_{\mathscr{O}}[E(\Lambda)+\Lambda E(M)-E(M)] d M \\
& =\int_{\mathscr{O}}[-E(M)+E(\Lambda M)] d M=0 .
\end{aligned}
$$

The last integral vanishes because it is an entire function of $\Lambda$ taking the value 0 when $\Lambda \in \mathcal{O}$ (by invariance of $d M$ ). Hence our problem is solved and, denoting $G^{ \pm}=F^{ \pm}-E_{0}$, there are constants $K$ and $V$, depending only on $U_{n}$ and $P_{n}$ such that

$$
\left\langle G^{+}, f\right\rangle \mid \leqq K N\left(f, \sigma^{\prime}, V, P_{n}\right) .
$$

This shows that, if the $T(X)$ have been defined in a Lorentz invariant way for $|X| \leqq n-1$, they can be defined in a Lorentz invariant way for $|X|=n$ (while still verifying inequalities of the type (22)).

\section{Conclusion}

It has been shown here that a renormalized perturbation series can be defined for a strictly localizable but non-polynomial Lagrangian. The requirements of locality and Lorentz invariance are fulfilled. The existence of an adiabatic limit for Green functions can be proved (for theories with non-zero masses) in the same way as in the polynomial case [1]. However, our treatment is preliminary since it does not touch on the question of minimality studied in Ref. [6]. Moreover the existence of a strong adiabatic limit remains to be proved.

Acknowledgements. The authors wish to thank Profs. J. Bros, K. Hepp, A. Jaffe, H. Lehmann, K. Pohlmeyer, and R. Stora for useful discussions. 


\section{References}

1. Epstein, H., Glaser, V.: CERN preprint TH 1156 reprinted in Vol. 11 of "Prépublications de la RCP no 25" (Strasbourg 1970) and to appear in the proceedings of the 1970 Les Houches Summer School. Also: CERN preprint TH 1400 to appear.

2. Jaffe, A.: Phys. Rev. 158, 1454 (1967); also: Contribution to the informal meeting on renormalization, ICTP, Trieste 1969.

3. Gelfand, I., Shilov, G.: Generalized functions, in particular, Vol. 2. Moscow: Fismatgiz, 1958.

4. Mandelbrojt, S.: Fonctions entières et transformées de Fourier et applications. Math. Soc. Jap. 1967.

5. Efimov, G.: Commun. math. Phys. 5, 42 (1967); 7, 138 (1968).

6. Lehmann, H., Pohlmeyer, K.: Contribution to the meeting on renormalization ICTP, Trieste 1969. Commun. math. Phys. 20, 101, 1971.

H. Epstein

I.H.E.S.

F-91 Bures-surYvette/France
V. Glaser

CERN

Geneva, Switzerland 